Check for updates

Cite this: Chem. Sci., 2017, 8, 7834

\title{
Harnessing fungal nonribosomal cyclodepsipeptide synthetases for mechanistic insights and tailored engineering $\dagger$
}

\author{
Charlotte Steiniger, ${ }^{a}$ Sylvester Hoffmann, ${ }^{a}$ Andi Mainz, ${ }^{a}$ Marcel Kaiser, ${ }^{\text {bc }}$ \\ Kerstin Voigt, ${ }^{d}$ Vera Meyer ${ }^{\mathrm{e}}$ and Roderich D. Süssmuth (D) *a
}

\begin{abstract}
Nonribosomal peptide synthetases represent potential platforms for the design and engineering of structurally complex peptides. While previous focus has been centred mainly on bacterial systems, fungal synthetases assembling drugs like the antifungal echinocandins, the antibacterial cephalosporins or the anthelmintic cyclodepsipeptide (CDP) PF1022 await in-depth exploitation. As various mechanistic features of fungal CDP biosynthesis are only partly understood, effective engineering of NRPSs has been severely hampered. By combining protein truncation, in trans expression and combinatorial swapping, we assigned important functional segments of fungal CDP synthetases and assessed their in vivo biosynthetic capabilities. Hence, artificial assembly line components comprising of up to three different synthetases were generated. Using Aspergillus niger as a heterologous expression host, we obtained new-to-nature octa-enniatin $\left(4 \mathrm{mg} \mathrm{L}^{-1}\right)$ and octa-beauvericin $\left(10.8 \mathrm{mg} \mathrm{L}^{-1}\right)$, as well as high titers of the hybrid CDP hexa-bassianolide $\left(1.3 \mathrm{~g} \mathrm{~L}^{-1}\right)$ with an engineered ring size. The hybrid compounds showed up to 12-fold enhanced antiparasitic activity against Leishmania donovani and Trypanosoma cruzi compared to the reference drugs miltefosine and benznidazole, respectively. Our findings thus contribute to a rational engineering of iterative nonribosomal assembly lines.
\end{abstract}

Received 14th July 2017

Accepted 25th September 2017

DOI: $10.1039 / c 7 s c 03093 b$

rsc.li/chemical-science

\section{Introduction}

Nonribosomal peptide synthetases (NRPSs) assemble a great variety of linear and cyclic peptides, which display a diverse spectrum of valuable pharmacologically useful bioactivities. Amongst those biosynthetic machineries, iterative-type NRPSs from filamentous fungi are a remarkable subgroup, which produce $N$-methylated cyclodepsipeptides (CDPs) of different ring sizes. Hexa-CDPs such as enniatin B (Fusarium ssp.) and beauvericin (Beauveria bassiana) as well as octa-CDPs like PF1022A (Rosellinia sp.) and bassianolide (B. bassiana) (Fig. 1b) show various antibacterial, anticancer, anthelmintic and insecticidal activities. ${ }^{1}$ This turns them into promising study

${ }^{a}$ Fachgebiet Biologische Chemie, Institut für Chemie, Technische Universität Berlin, Strasse des 17. Juni 124, 10623 Berlin, Germany. E-mail: roderich.suessmuth@ tu-berlin.de

${ }^{b}$ Parasite Chemotherapy, Medical Parasitology \& Infection Biology, Swiss Tropical and Public Health Institute, Socinstrasse 57, 4051 Basel, Switzerland

'University of Basel, Petersplatz 1, 4003 Basel, Switzerland

${ }^{d}$ Jena Microbial Resource Collection (JMRC), Leibniz-Institut für Naturstoff-Forschung und Infektionsbiologie, Hans-Knöll-Institut, Adolf-Reichwein-Straße 23, 07745 Jena, Germany

${ }^{e}$ Fachgebiet Angewandte und Molekulare Mikrobiologie, Institut für Biotechnologie, Technische Universität Berlin, Gustav-Meyer-Allee 25, 13355 Berlin, Germany

$\dagger$ Electronic supplementary information (ESI) available: Detailed methodology, alignments, cloning, purification, NMR, bioactivity. See DOI: 10.1039/c7sc03093b candidates for further drug discovery and development. Iterative CDP synthetases consist of two modules, each containing domains for adenylation (A), transfer (T) and condensation (C) of the substrate, as well as a terminal $\mathrm{T}_{2 \mathrm{~b}}-\mathrm{C}_{3}$ bidomain (Fig. 1a). The first module $\left(\mathrm{M}_{1}\right)$ activates $\mathrm{D}$ - $\alpha$-hydroxycarboxylic acids (D-HA) and has been shown to be promiscuous concerning aliphatic and aromatic D-HAs to varying extents. ${ }^{2-4}$ The D-HA is subsequently coupled to an $\mathrm{L}$ - $\alpha$-amino acid (L-AA), which is activated and $N$-methylated by the second module $\left(\mathrm{M}_{2}\right)$ containing an auxiliary $N$-methyltransferase (Mt) domain. Module 2 has also been reported to process different $N$-Me-L-amino acid ( $N$-Me-L-AA) building blocks to a certain degree., ${ }^{5,6}$ Concerning substrate elongation, it has been proposed that the growing depsipeptide chain is elongated either in dipeptidol units (parallel mode $)^{7,8}$ or in a stepwise fashion using single HA or AA building blocks (linear mode; Fig. 1a). ${ }^{9}$ Recently, the linear mode has been strongly favored, stating that the growing chain is shuttling between the $\mathrm{T}_{1}$ and $\mathrm{T}_{2 \mathrm{a} / \mathrm{b}}$ domains which alternately switch their donor and acceptor roles. ${ }^{10}$ For efficient macrocyclization of the full-length linear depsipeptide precursor, the $\mathrm{N}$-terminal $\mathrm{C}_{1}$ and $\mathrm{C}$-terminal $\mathrm{C}_{3}$ domain have previously been proposed to interact by forming a cavern. ${ }^{9}$ In contrast, the $\mathrm{C}_{3}$ domain alone was very recently found to mediate macrocyclization, ${ }^{10}$ which is in agreement with the established function of so-called termination $\mathrm{C}\left(\mathrm{C}_{\text {term }}\right)$ domains in fungal linear NRPSs. ${ }^{11}$ Still, various biosynthetic features of CDP synthetases 
a
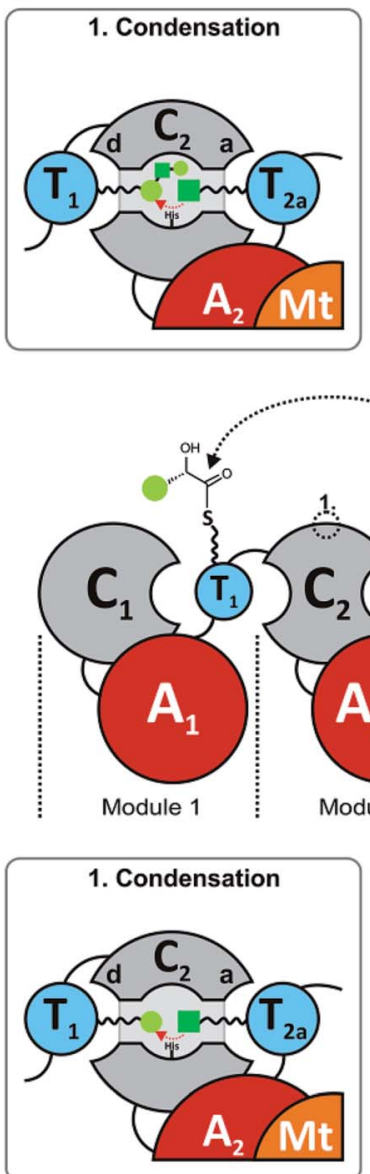

Looping Model
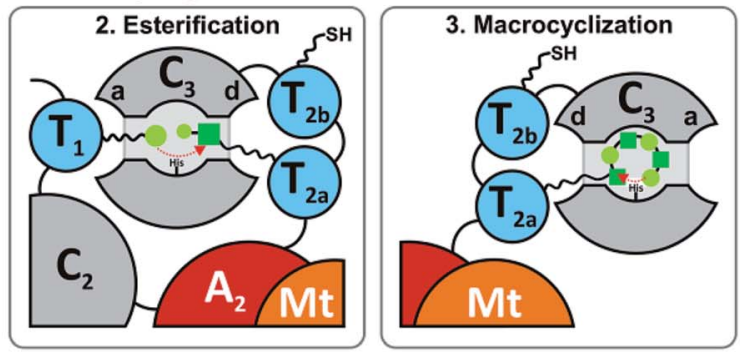

0

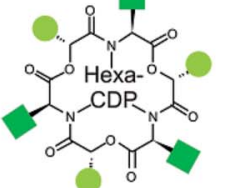

Čnain elongation Macrocyclization

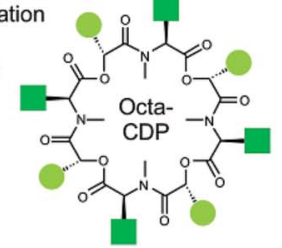

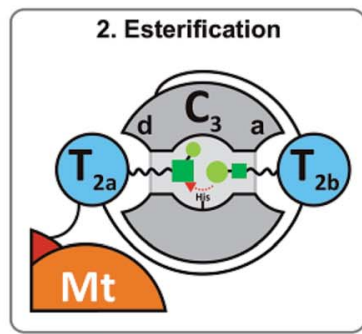

Parallel Model

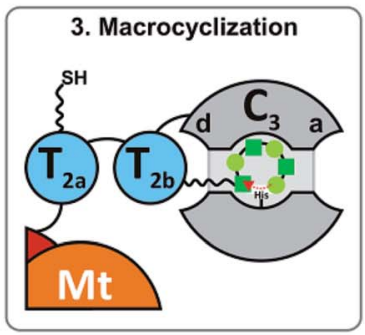

b
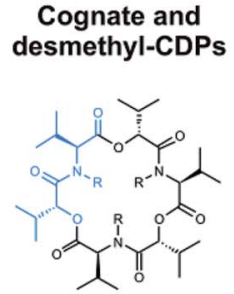

Enniatin $\mathrm{B} \mathrm{R}=\mathrm{CH}_{3}$ Desmethyl-enniatin B $\mathrm{R}=\mathrm{H}$

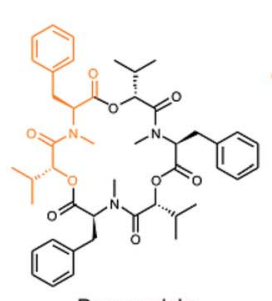

Beauvericin

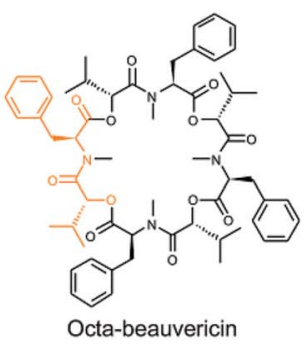

Hybrid CDPs

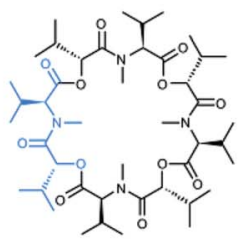

Octa-enniatin B
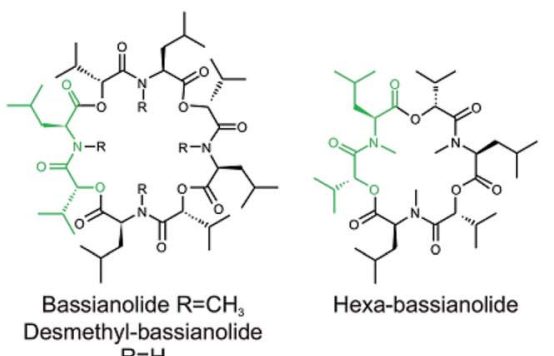

Hexa-bassianolide

Fig. 1 Alternative CDP biosynthesis models and structures. (a) Models of CDP biosynthesis. Fungal CDP synthetases produce six- and eightmembered CDPs, with module 1 incorporating D-HAs and module 2 activating L-AAs. The building blocks are coupled until the chain length is long enough to be cyclized by the $\mathrm{C}_{3}$ domain. In the looping model (top), the depsipeptide chain grows by attachment of a single building block ( $\mathrm{HA}$ or $\mathrm{AA}$ ), shuttling between $\mathrm{T}_{1}$ and $\mathrm{T}_{2 \mathrm{a} / \mathrm{b}}$. The role of $\mathrm{T}_{2 \mathrm{~b}}$ is still unclear. In the parallel model (bottom), the depsipeptide chain grows by addition of dipeptidols. a: acceptor site, d: donor site. (b) Cognate, non-methylated and hybrid CDP products. Iterative units are colored.

are only partially understood, including mechanistic insights into how hexa- and octa-CDPs are assembled.

Due to their modular architecture and high homology, CDP synthetases appear well suited to study domain-specific contributions by combinatorial exchanges, while generating novel peptide structures. Consequently, fungal NRPSs offer an excellent opportunity for engineering approaches. Inspiration comes from various engineering attempts in bacterial systems, including module fusion, ${ }^{12}$ substitution, ${ }^{13}$ extension ${ }^{14}$ and deletion ${ }^{15}$ of NRPS segments. Initial attempts in fungal NRPSengineering have shown encouraging results in vivo, leading to novel nonribosomal peptides (NRPs) including derivatives of enniatin and beauvericin with altered D-HA side chains. ${ }^{16-18}$ Since the chemical synthesis of CDPs has some drawbacks and limitations, biotechnological production in heterologous microbial cell factories is a promising alternative for sustainable CDP production. ${ }^{17,19}$ However, as long as our mechanistic understanding of fungal NRPS assembly remains limited, an effective engineering of fungal iterative NRPSs is unfeasible. We thus focused on the functional analysis of various catalytic steps of CDP synthetases, whilst concomitantly establishing an in vivo expression system for new-to-nature CDP derivatives with altered bioactivities. While we were preparing this manuscript, Yu et al. proposed a linear CDP biosynthesis model, which complements our findings. ${ }^{10}$

\section{Experimental}

\section{Selection and design of swapping and truncation sites}

Structural information about flexible $\mathrm{T}-\mathrm{C}$ linker regions was obtained from the $\mathrm{T}_{5} \mathrm{C}_{6}$ bidomain of tyrocidine synthetase from Brevibacillus brevis $\left(\mathrm{TycC}_{5-6}\right.$; PDB 2JGP). ${ }^{20}$ Using the $\mathrm{TycC}_{5-6} \mathrm{~T}-\mathrm{C}$ bidomain as a template, structural models of EnSYN-, BeSYN- and BaSYN- $\mathrm{T}_{1}-\mathrm{C}_{2}$ and $\mathrm{T}_{2 \mathrm{~b}}-\mathrm{C}_{3}$ bidomains were generated (SWISSMODEL $\left.{ }^{21}\right)$. The homology models were aligned to $\mathrm{TycC}_{5-6}$ (PyMOL, Schrödinger, LLC) to identify promising swapping sites in the linker regions. The structurally identified swapping regions were annotated in a multiple sequence alignment with the synthetases PF-, En-, Be- and BaSYN in order to define the distinct $\mathrm{T}_{1}-\mathrm{C}_{2}$ and $\mathrm{T}_{2 \mathrm{~b}}-\mathrm{C}_{3}$ swapping sites (ESI Fig. $1 \dagger$ ). The latter site was 
also used for the construction of $\operatorname{EnSYN} \Delta \mathrm{C}_{3}$ (ESI Fig. $2 \dagger$ ). For $\mathrm{T}_{2 \mathrm{a}}-\mathrm{T}_{2 \mathrm{~b}}$ swapping, the determined $\mathrm{T}_{2 \mathrm{~b}}-\mathrm{C}_{3}$ swapping site was annotated for the $\mathrm{T}_{2 \mathrm{a}}-\mathrm{T}_{2 \mathrm{~b}}$ linker region in a multiple sequence alignment. Structural information about the $\mathrm{C}_{3}$ domain was obtained from the $\mathrm{T}-\mathrm{C}_{\text {term }}$ bidomain of TqaA from Penicillium aethiopicum (PDB 5EJD, ESI Fig. $3 \dagger$ ). ${ }^{22}$ Based on TqaA- $\mathrm{C}_{\text {term }}$, the borders of the two subdomains $\mathrm{C}_{\mathrm{NTD}}$ and $\mathrm{C}_{\mathrm{CTD}}$ were defined (ESI Fig. $4 \dagger$ ) and annotated in a multiple sequence alignment with PF-, En-, Be- and BaSYN (ESI Fig. $5 \dagger$ ) in order to define the distinct

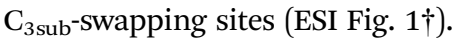

For construction of the $\mathrm{SYN} \Delta \mathrm{C}_{1}$ constructs, structural information about the $\mathrm{C}-\mathrm{A}$ linker region was obtained from the termination module (C-A-T-Te) of surfactin A-synthetase from Bacillus subtilis (SrfA-C; PDB 2VSQ). ${ }^{23}$ The identified truncation region was annotated in a multiple sequence alignment with $\mathrm{PF}$-, En-, Be- and BaSYN to define the distinct $\mathrm{C}_{1}$ truncation site (ESI Fig. $2 \dagger$ ). For truncation of the Mt domain insertion, the boundaries were identified in a multiple sequence alignment of the PF-, En-, Be- and BaSYN-A $\mathrm{A}_{1}$ and $-\mathrm{A}_{2}$ domains as well as the gramicidin A synthetase A domain (GrsA). ${ }^{24}$ Structural information about A domains was obtained from the crystal structure of GrsA (PDB 1AMU) ${ }^{24}$ to identify secondary structure elements flanking the loop region in which Mt domains are commonly embedded. The loop region was annotated in a multiple sequence alignment of the PF-, En-, Be- and BaSYN-A $\mathrm{A}_{2}$ domains to identify the distinct truncation sites (ESI Fig. $2 \dagger$ ). Based on GrsA, structural models of the EnSYN- and BaSYN-A $A_{1}$ domain and truncated $\mathrm{A}_{2}$ domains were generated (SWISS-MODEL) and aligned (PyMOL) to confirm the construction of a loop in $\mathrm{A}_{2}$ similar to $\mathrm{A}_{1}$ (ESI Fig. $6 \dagger$ ). Further experimental details are provided in the ESI. $\dagger$

\section{Results and discussion}

\section{Providing the basis for an efficient fungal NRPS engineering}

Due to low synthesis yields, many engineered in vivo NRPS systems are of limited value for deriving extensive conclusions on mechanisms or for a sustainable production..$^{13,14,17}$ In order to lay a robust foundation for engineering approaches, we investigated the individual contributions of the $\mathrm{C}_{1}, \mathrm{C}_{3}, \mathrm{~T}_{2 \mathrm{a} / \mathrm{b}}$ and Mt domains to CDP biosynthesis (Fig. 1a). A comparison of the seven conserved core motifs of canonical $\mathrm{C}$ domains ${ }^{25}$ indicated that they all differ significantly between the $\mathrm{C}_{1}, \mathrm{C}_{2}$ and $\mathrm{C}_{3}$ domains, suggesting different roles in CDP biosynthesis (ESI Tables $1-3 \dagger$ ). This inspired us to construct truncated versions of the wild-type enniatin synthetase (EnSYN), beauvericin synthetase (BeSYN) and bassianolide synthetase (BaSYN) lacking the $\mathrm{C}_{1}$ domain. All expression assays were performed in vivo in Escherichia coli (DE3), harboring the fungal phosphopantetheinyl transferase (PPTase) $n p g A$ derived from $A$. nidulans DM3365 on a second plasmid. The co-expressed NpgA displayed a 14-fold enhanced production of the fungal CDP products in comparison to the endogenous PPTase and 4.8-fold increase when compared to Sfp (ESI Fig. $7 \dagger$ ). Crude ethyl acetate extracts of the cell pellets were analyzed by MALDI-TOF-MS and LC-ESI-MS. Unlike previous approaches in Saccharomyces cerevisiae, ${ }^{\mathbf{1 0}}$ the GST-tagged $\mathrm{C}_{1}$ deletion constructs $\left(\mathrm{SYN} \Delta \mathrm{C}_{1}\right.$; $\sim 324 \mathrm{kDa}$ ) were successfully produced in $E$. coli co-expressing NpgA (ESI Fig. 8†). Most importantly, all SYN $\Delta \mathrm{C}_{1}$ constructs maintained production of their cognate CDPs (Fig. 2a), while production yields dropped to $1-55 \%$ compared to the wild-type enzymes (Fig. 2c). This clearly indicated that the $\mathrm{C}_{1}$ domain plays an important role in CDP assembly, but that, in contrast to the cavern model, ${ }^{9}$ the $\mathrm{C}_{1}$ domain is not involved in macrocyclization.

In accordance with a recent in trans expression of the BeSYN$\mathrm{C}_{3}$ domain, ${ }^{10}$ we generated truncated versions of EnSYN lacking the $\mathrm{C}_{3}$ domain (EnSYN $\Delta \mathrm{C}_{3}$ ) and monitored in vivo production of enniatins with and without co-expression of the excised EnSYN-C domain. EnSYN $\Delta \mathrm{C}_{3}$ was unable to produce enniatins, however, CDP production could be restored in trans by co-expression with the free-standing EnSYN-C ${ }_{3}$ domain (Fig. 2b). The EnSYN-C domain could also restore enniatin production of a synthetase construct devoid of both the $\mathrm{C}_{1}$ and $\mathrm{C}_{3}$ domains (EnSYN $\Delta \mathrm{C}_{1} \mathrm{C}_{3}$; Fig. 2b). Surprisingly, a combination of $\operatorname{EnSYN} \Delta\left(\mathrm{C}_{1}\right) \mathrm{C}_{3}$ with a heterologous $\mathrm{C}_{3}$ domain derived from the octa-CDP-producing BaSYN even enabled the production of new-to-nature octa-enniatin B (comprising one D-Hiv-Val unit more; Fig. 2b). To the best of our knowledge, octa-enniatin has not been reported to date. Hence, the function of the $\mathrm{C}_{3}$ domain can clearly be assigned to macrocyclization and ring size determination, which supports recent findings. ${ }^{10}$ Additionally, we aimed at challenging the flexibility of fungal CDP synthetases concerning nonmethylated AAs. Therefore, we constructed variants of EnSYN and BaSYN devoid of the Mt domain (EnSYN $\Delta$ Mt, BaSYN $\Delta$ Mt; ESI Fig. $6 \dagger$ ). Indeed, EnSYN $\Delta$ Mt and BaSYN $\Delta$ Mt exclusively produced the non-methylated analogues desmethyl-enniatin and desmethyl-bassianolide, respectively (Fig. 2a). Notably, these compounds represent the first example of a directed in vivo production of CDPs lacking backbone methylation. The findings are reminiscent of natural enniatins produced by EnSYN with only one or two $N$-methylations (type B2, B3), while for BaSYN, no natural non-methylated species have been reported so far. Finally, we challenged the linear CDP elongation model similar to a recent BeSYN-based approach ${ }^{\mathbf{1 0}}$ by constructing versions of EnSYN with mutations at the conserved serines bearing the phosphopantetheine groups Ppant $2 \mathrm{a}$ (S2538A) or Ppant ${ }_{2 \mathrm{~b}}$ (S2632A). Like the BeSYN-variants, both mutants (EnSYN $\Delta$ Ppant $_{2 \mathrm{a}}$, EnSYN $\Delta$ Ppant $_{2 \mathrm{~b}}$ ) were still able to produce their wild-type CDP enniatin in vivo in E. $\operatorname{coli}$ (Fig. 2b). Similar to recent findings, ${ }^{10}$ the production of enniatin $\mathrm{B}$ by EnSYN $\Delta$ Ppant $_{2 \mathrm{a}}$ dropped to $0.4 \%$, whereas

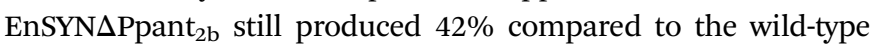
synthetase (Fig. 2c). This observation confirms that the use of $\mathrm{T}_{2 \mathrm{a}}$ or $\mathrm{T}_{2 \mathrm{~b}}$ as a waiting position is very unlikely, thus strongly supporting the linear/looping model.

\section{Active chain-length control for hybrid CDP production}

The identification of the $\mathrm{C}_{3}$ domain as the ring size determining domain in fungal iterative NRPSs ${ }^{\mathbf{1 0}}$ inspired us to design new-tonature CDPs of tailored ring size. To this end, we generated a set of hybrid synthetases based on the hexa-CDP-producing EnSYN and BeSYN as well as the octa-CDP producing BaSYN by exchanging the $\mathrm{C}_{3}$ domain or the $\mathrm{T}_{2 \mathrm{~b}}-\mathrm{C}_{3}$ bidomain. This rendered $\mathrm{XSYN}^{-\mathrm{YC}_{3}}$ and $\mathrm{XSYN}-\mathrm{YTC}_{3}$ constructs with $\mathrm{X}$ and $\mathrm{Y}$ referring to the NRPS core and the complementary heterologous 
a
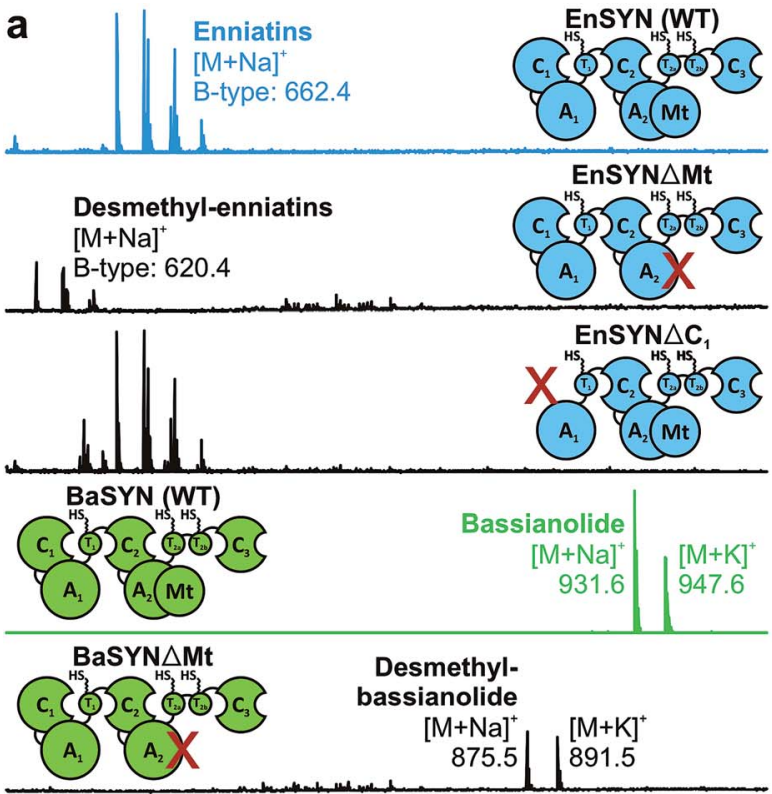

BaSYN $\triangle C$

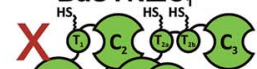

(A) $\left(A_{2}, M\right.$

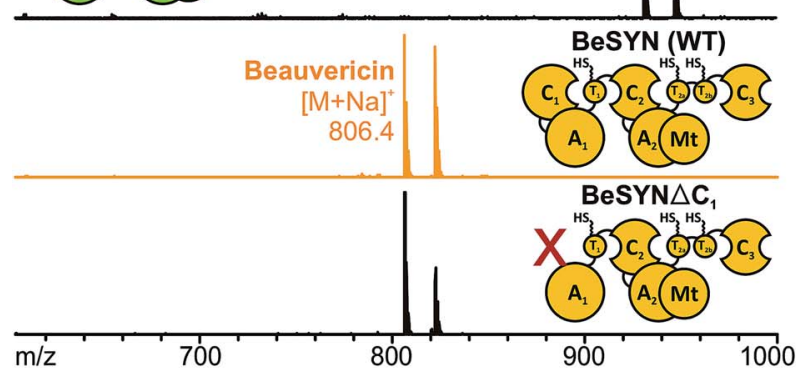

b Enniatins

$[\mathrm{M}+\mathrm{Na}]^{+}$

B-type: 662.4
EnSYN (WT)

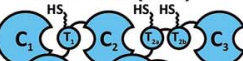

$\left(\mathrm{A}_{1} \mathrm{~A}_{2} \mathrm{Mt}\right.$

EnSYN $\triangle$ Ppant ${ }_{2 a}$

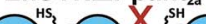

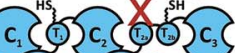

A $A_{1}, \mathrm{Mt}_{1}$

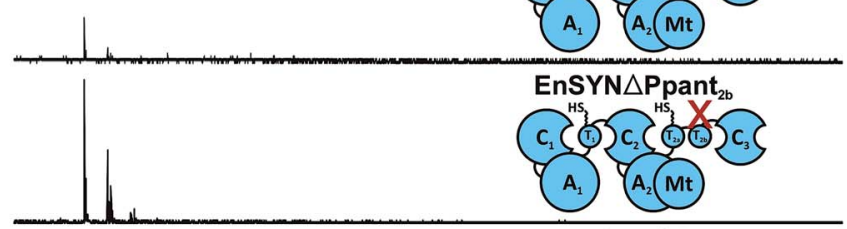

EnSYN $\triangle C$

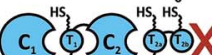

(A.) $A_{2}, M t$

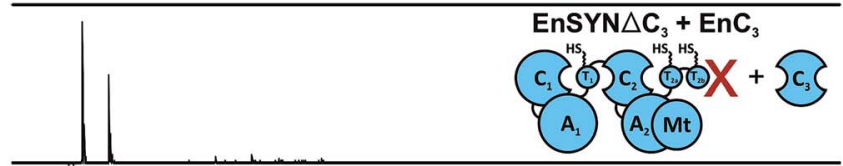

EnSYN $\triangle C_{1} C_{3}+E_{3} C_{3}$
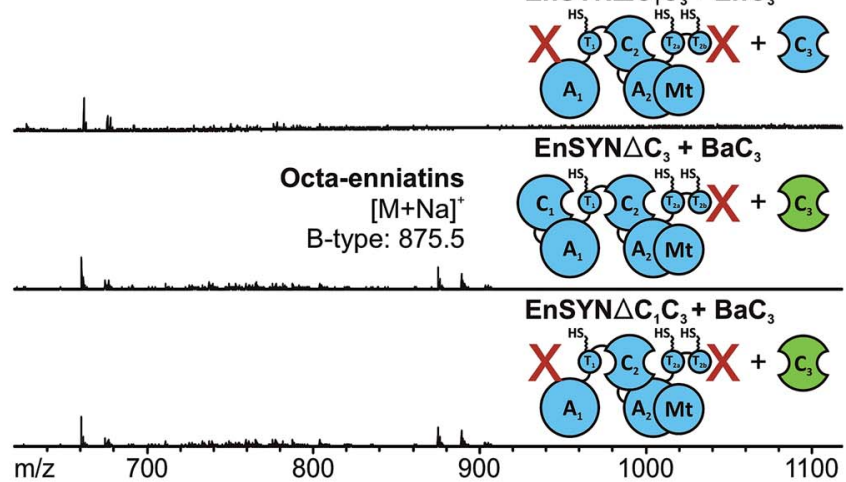

C
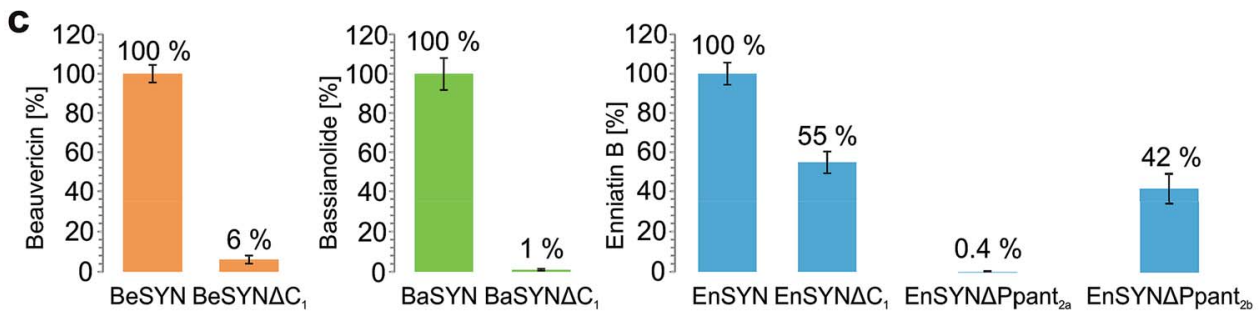

Fig. 2 Mechanistic studies on fungal CDP synthetases. In vivo CDP production of (a) wild-type, mutated (EnSYN $\Delta$ Ppant $2 a / b)$ and truncated synthetases (SYN $\Delta \mathrm{C}_{1}$, SYN $\Delta \mathrm{Mt}$ ) as well as (b) in trans combinations synthesized in E. coli and monitored by MALDI-TOF-MS. (c) CDP yields in E. coli strains containing wild-type, $\Delta \mathrm{C}_{1}$ - and $\Delta \mathrm{Ppant}_{2 \mathrm{a} / \mathrm{b}}$-synthetases determined by LC-ESI-MS ( $n=3$ cultures, standard deviation).

segment, respectively (Fig. 3). Based on the identified domain boundaries in multiple sequence and structure alignments (see Experimental section), the domain swapping was performed in the inter-domain linker regions (ESI Fig. $1 \dagger$ ). The resulting GSTtagged hybrid synthetases $(\sim 375 \mathrm{kDa})$ were produced in soluble form in E. coli co-expressing NpgA (ESI Fig. $8 \dagger$ ). Indeed, the hybrid synthetases EnSYN-BaTC 3 and EnSYN- BaC $_{3}$ both efficiently produced octa-enniatin B $(4 \times N$-Me-L-Val, $m / z 853.553$ $[\mathrm{M}+\mathrm{H}]^{+}$; Fig. 3a). In analogy, the hybrids BeSYN-BaTC ${ }_{3}$ and BeSYN-BaC 3 produced octa-beauvericin $(m / z) 1045.553[\mathrm{M}+\mathrm{H}]^{+}$; Fig. 3c). So far, octa-beauvericin was only known from total synthesis ${ }^{26}$ and the in vivo biosynthesis has been reported very recently. ${ }^{10}$ Closer inspection of the biosynthesis products revealed that the octa-CDP producing hybrid synthetases EnSYN-Ba(T) $\mathrm{C}_{3}$ and BeSYN-Ba(T) $\mathrm{C}_{3}$ still produced trace amounts of the natural smaller ring size (Fig. 3a and c). Notably, the wild-type BaSYN is also able to produce minor amounts of hexa-bassianolide (Fig. 5b). Therefore, we can conclude that the BaSYN- $\mathrm{C}_{3}$ domain also cyclizes the premature CDP chain, which is in contrast to recent statements. ${ }^{10}$ The four BaSYN-based hybrid constructs BaSYN-En(T) $\mathrm{C}_{3}$ and BaSYN-Be(T) $\mathrm{C}_{3}$ produced the respective hybrid CDP hexa-bassianolide (corresponding to enniatin C, $3 \times N$-Me-L-Leu, $m / z 682.463[\mathrm{M}+\mathrm{H}]^{+}$; Fig. 3b). Apart from BaSYN, hexa-bassianolide is also a natural 
a
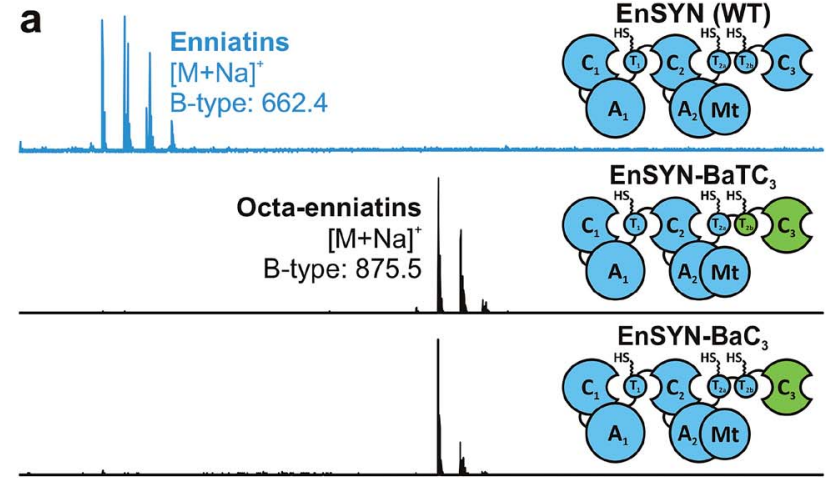

C $\operatorname{BeSYN}($ WT)
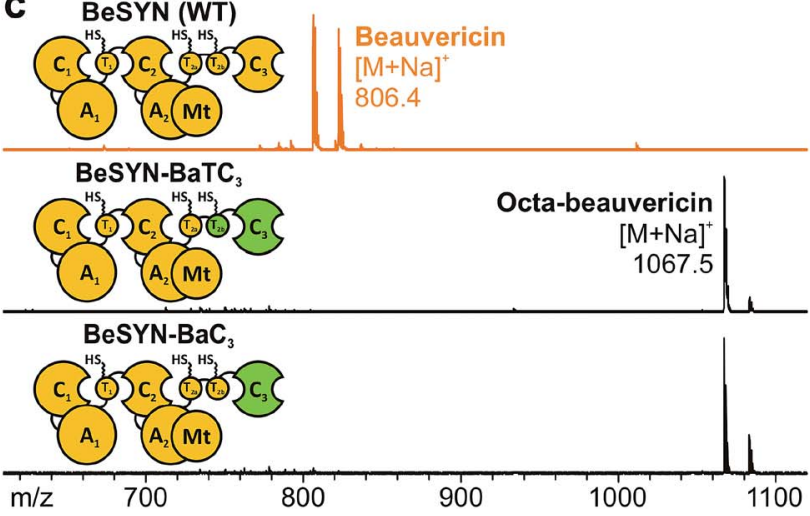

$\operatorname{EnSYN~(WT)~}$

$A_{1}$

$\mathrm{A}_{1} \mathrm{~A}_{2} \mathrm{Mt}$

EnSYN-BaTC

EnSYN-BaC

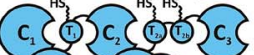

b

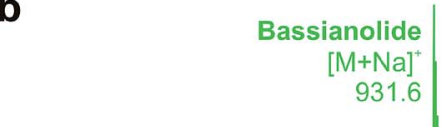

931.6

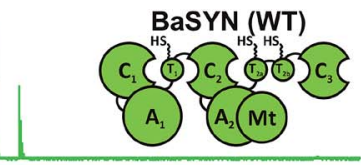

BaSYN-EnTC
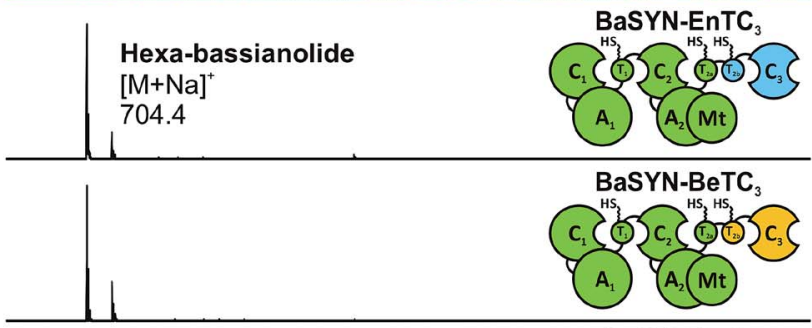

$\mathrm{BaSYN}-\mathrm{BeC}_{3}$

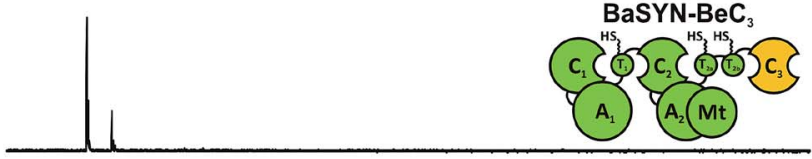

BaSYN-EnC

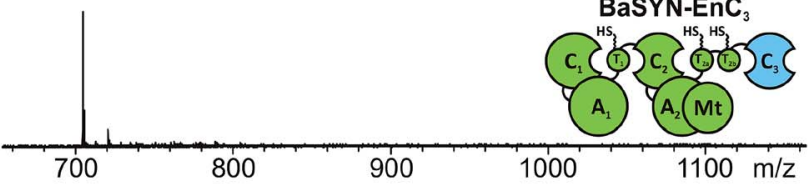

Fig. 3 Structural diversification of fungal CDPs. In vivo CDP production of wild-type and hybrid synthetases with swapped C-terminal part (see color code) based on EnSYN (a), BaSYN (b) and BeSYN (c) synthesized in E. coli and monitored by MALDI-TOF-MS.

side product of the wild-type EnSYN. ${ }^{27}$ The results reinforce the control function of the $\mathrm{C}_{3}$ domain with respect to the encrypted chain length for macrocyclization as well as its substrate flexibility concerning aliphatic and aromatic $N$-Me-L-AAs.

\section{A detailed view of the $\mathrm{C}_{3}$ domain}

In analogy to conventional elongation $\mathrm{C}$ domains $\left(\mathrm{C}_{\text {elong }}\right)$ and fungal $\mathrm{C}_{\text {term }}$ domains, ${ }^{20,22,28}$ the $\mathrm{C}_{3}$ domain consists of an $\mathrm{N}$ - and C-terminal subdomain $\left(\mathrm{C}_{\mathrm{NTD}}, \mathrm{C}_{\mathrm{CTD}}\right)$, which together form a V-shaped pseudo-dimer with the active site buried in the cleft between the two subdomains (ESI Fig. $3 \dagger$ ). Whereas $\mathrm{C}_{\mathrm{NTD}}$ harbors the catalytically relevant $\mathrm{His}_{\mathrm{cat}}, \mathrm{C}_{\mathrm{CTD}}$ provides most of the interface residues towards the donor $\mathrm{T}$ domain. In contrast to the $\mathrm{C}_{\mathrm{term}}$ domain of the fungal tryptoquialanine (TqaA) synthetase that is closed at its acceptor site, ${ }^{22}$ the $\mathrm{C}_{3}$ domain of fungal CDP synthetases also catalyzes condensation reactions. ${ }^{10}$ Hence, the $\mathrm{C}_{3}$ domain features a functional acceptor site competent to catalyze ester bond formation. In order to gain insights into the molecular determinants of macrocyclization and ring size control by the $\mathrm{C}_{3}$ domain, we started with a sequence-based shuffling approach. An alignment of the $\mathrm{C}_{3}$ domains revealed ten residues potentially involved in ring size determination (ESI Fig. $5 \dagger$ ). When visualized in a superposition of the TqaA- $\mathrm{C}_{\text {term }}$ structure ${ }^{22}$ with a structural model of EnSYN-C ${ }_{3}$ (ESI Fig. $9 \dagger$ ), five from the originally ten conspicuous residues remained, which were swapped from the BaSYN to the EnSYN sequence (EnSYN-mutC ${ }_{3}$ ). Exchanging these five amino acids did not hamper CDP production, but did not lead to an altered ring size either (Fig. 4a), ruling out these residues in terms of ring size control.

In an alternative approach, we swapped the subdomains $\mathrm{C}_{3 \mathrm{NTD}}$ and $\mathrm{C}_{3 \mathrm{CTD}}$ of BaSYN into the EnSYN and BeSYN system (ESI Fig. $4 \dagger$ ). Expression of the four constructs EnSYN-BaC ${ }_{3 \mathrm{NTD}}$, EnSYN-BaC 3 CTD, BeSYN-BaC ${ }_{3 N T D}$ and BeSYN-BaC 3 CTD in $E$. coli co-expressing NpgA showed that CDP production was not hampered by the extensive heterologous intra-domain interfaces (Fig. 4). Remarkably, a swap of $\mathrm{C}_{3 \mathrm{CTD}}$ gave rise to minor production of octa-CDPs. In the EnSYN-based system, a swap of $\mathrm{C}_{3 \mathrm{NTD}}$ even slightly enhanced production of octa-enniatin B (Fig. 4a), whereas only trace amounts of octa-beauvericin could be observed for BeSYN-BaC ${ }_{3 \mathrm{NTD}}$ (Fig. $4 \mathrm{~b}$ ). This data encouraged an additional swap of the bridging loop in $\mathrm{C}_{3 \mathrm{CTD}}$ (EnSYN-BaC ${ }_{3 N T D+l o o p}$, EnSYN-Ba loop), the region which crosses over to $\mathrm{C}_{3 \mathrm{NTD}}$ and forms a lid above the cyclization pocket (ESI Fig. $3 \dagger$ ). However, swapping of the bridging loop completely abolished CDP production (Fig. 4a), suggesting that the conformation of the $\mathrm{C}_{3}$ domain has been massively disturbed. Thus, we hypothesize that CDP ring size is controlled not only by the size of the substrate pocket, but also by the acceptor- and donor-site interfaces that mediate the recruitment and correct positioning of the corresponding partner $\mathrm{T}$ domains. Such regulation via the $\mathrm{T}-\mathrm{C}$ interfaces becomes in particular relevant in light of the recently postulated linear mode of CDP synthetases, ${ }^{10}$ which relies on a competition between $\mathrm{T}_{1}$ and $\mathrm{T}_{2 \mathrm{a} / \mathrm{b}}$ for binding to $\mathrm{C}_{3}$ leading either to chain elongation or macrocyclization. 


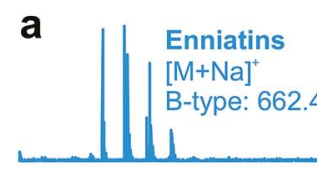

EnSYN (WT)
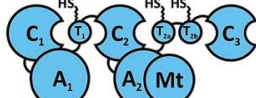

EnSYN-BaC ${ }_{3}$

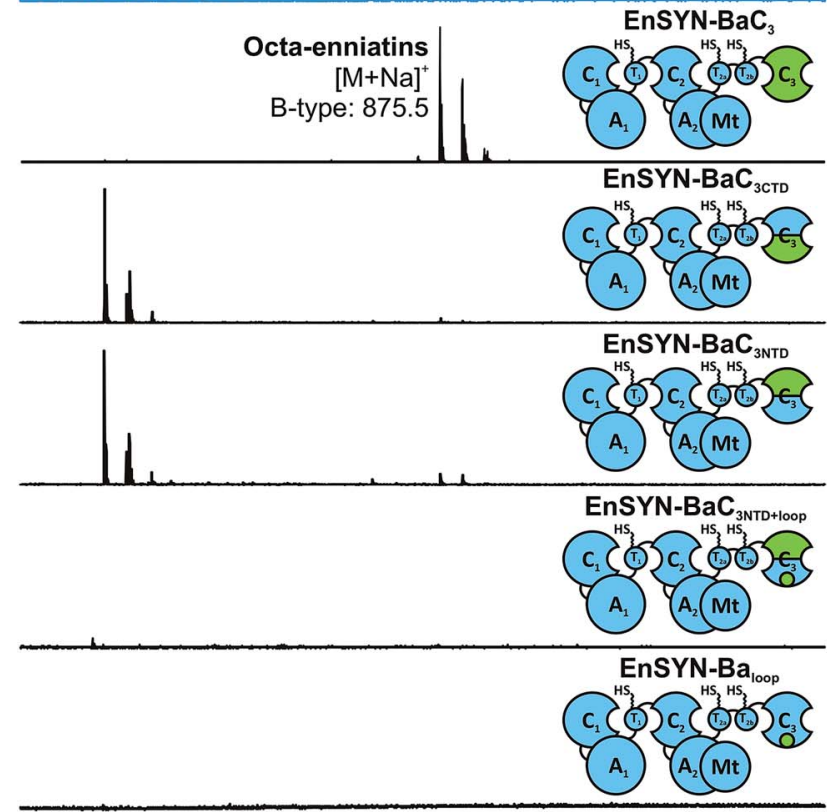

EnSYN-mutC ${ }_{3}$

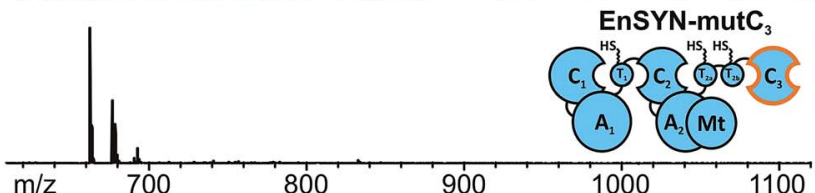

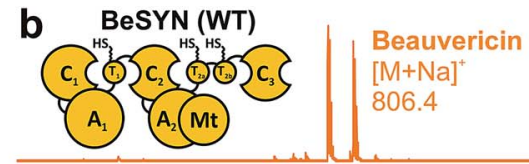

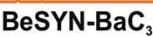

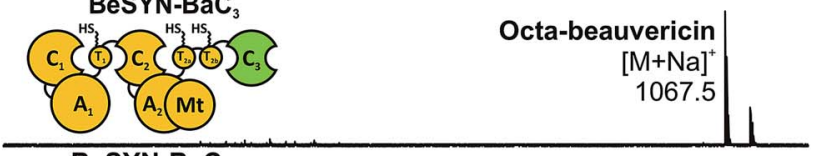

BeSYN-BaC

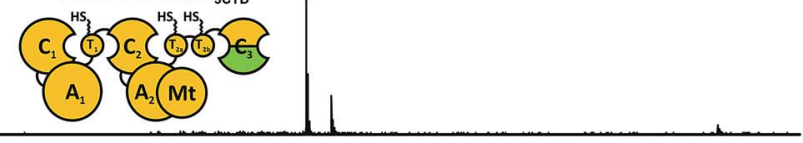

BeSYN-BaC ${ }_{\text {3NTD }}$

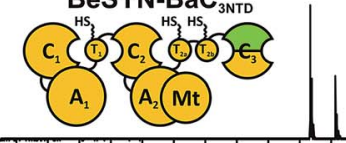

$\mathrm{m} / \mathrm{z}$

\section{0}

900

1000

1100

Fig. 4 Swapping of $\mathrm{C}_{3}$ subdomains and elements thereof. In vivo CDP production of wild-type and hybrid synthetases based on EnSYN (a) and BeSYN (b) with swapped $C_{3 N T D}, C_{3 C T D}$, bridging loop region (small green circle) and five distinct AAs of BaSYN (orange line) in E. coli, respectively, as monitored by MALDI-TOF-MS.

\section{Scale-up production in $A$. niger and bioactivities of hybrid CDPs}

In previous engineering approaches, the use of $E$. coli as a bacterial heterologous host proved valuable for rapid proof-of-principle approaches. ${ }^{17}$ However, the fungal heterologous host A. niger appears to be more suitable for scale-up of fungal CDP production, as it renders significantly higher CDP yields. ${ }^{17,19}$ Therefore, we aimed for an enhanced production of the hybrid CDPs octa-enniatin B, octa-beauvericin and hexa-bassianolide by using $A$. niger under control of an inducible expression system. $^{\mathbf{1 9}}$ For that purpose, we performed polyethylene glycol (PEG)-mediated transformation of two A. niger strains differing in their protease profiles (strains AB1.13 and MA169.4) with the hybrid synthetase genes of EnSYN-BaTC ${ }_{3}$,

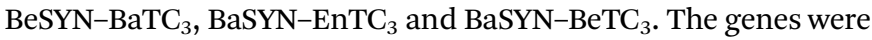
integrated into the fungal genome by homologous recombination (ESI Fig. 10†). From the two alternative constructs BaSYN-EnTC ${ }_{3}$ and BaSYN-BeTC ${ }_{3}$ synthesizing hexa-bassianolide, the former was chosen for scale-up in A. niger due to a higher production (ESI Fig. 11†).

All hybrid CDPs were purified by extraction with ethyl acetate, flash chromatography and reversed phase HPLC. Purification of the hybrid octa-CDPs from A. niger yielded $4 \mathrm{mg} \mathrm{L}^{-1}$ of octa-enniatin $\mathrm{B}$ and $10.8 \mathrm{mg} \mathrm{L}^{-1}$ of octa-beauvericin, respectively. Intriguingly, purification of hexa-bassianolide produced by BaSYN-EnTC ${ }_{3}$ afforded very high titers of approximately $1.3 \mathrm{~g} \mathrm{~L}^{-1}$. To our knowledge, this is the first report of an artificial NRPS producing on the $\mathrm{g} \mathrm{L}^{-1}$-scale, which underlines the great potential of fungal NRPS systems for biosynthetic engineering. Without understanding the catalytic assembly in detail, the higher titer in comparison to the hybrid octa-CDPs might be attributed to a more efficient interaction between the heterologous parts. For example, combining BaSYN with segments of EnSYN in E. coli rendered significantly higher titers than BaSYN-BeSYN combinations (Fig. 5b), which were also used for the production of octa-beauvericin (BeSYN-BaTC ${ }_{3}$ ). Since both wild-type BaSYN and EnSYN naturally produce minor amounts of hexa-bassianolide, the building blocks D-Hiv and L-Leu represent cognate substrates for both NRPS systems, thus ensuring efficient processing. To date, hexa-bassianolide could only be obtained in low amounts either from chemical synthesis, ${ }^{29}$ by precursor-directed biosynthesis $\left(2.2 \mathrm{mg} \mathrm{L}^{-1}\right)^{30}$ or from extraction of cultures of Verticillium hemipterigenum (BCC 1449; $\left.0.3 \mathrm{mg} \mathrm{L}^{-1}\right) .{ }^{27}$ In a very recent report on heterologous CDP production in $S$. cerevisiae, no yields were mentioned. ${ }^{10}$ 

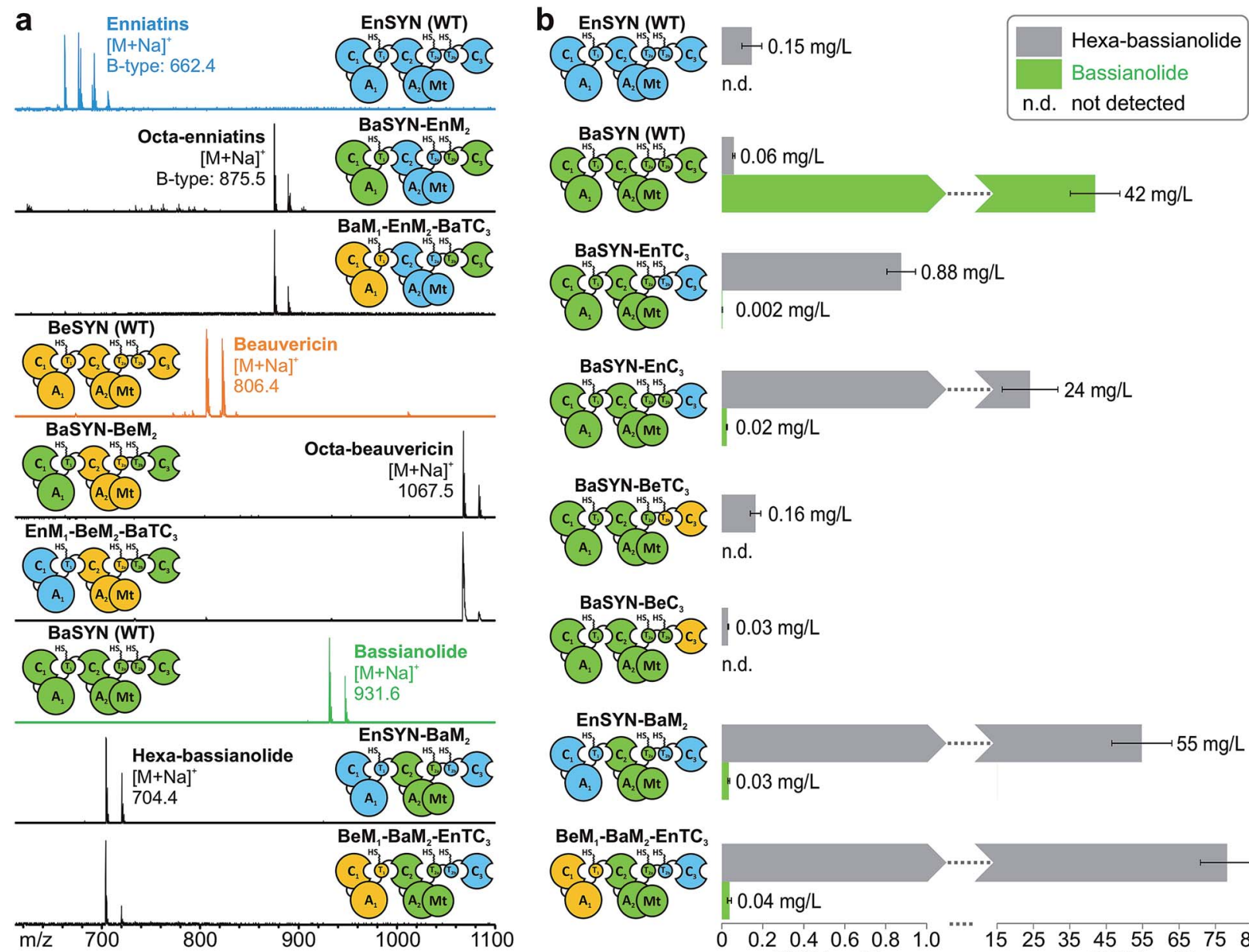

BaSYN-BeC

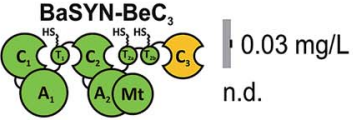

EnSYN-BaM
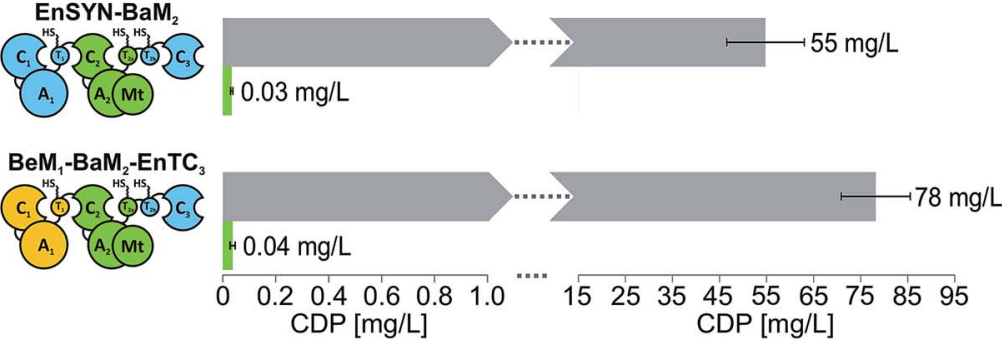

Fig. 5 Multiple swapping of fungal CDP synthetases. (a) In vivo CDP production of the hybrid synthetases (see color code) monitored by MALDITOF-MS. (b) CDP titers of wild-type and hybrid NRPSs producing bassianolide and hexa-bassianolide in E. coli compared by LC-ESI-MS ( $n=3$ cultures, standard deviation). n. d.: not detected.

Based on the sufficient amount of the hybrid CDPs for biological profiling and the broad activity range of CDPs, ${ }^{30-32}$ octa-enniatin B, octa-beauvericin and hexa-bassianolide were tested for antiparasitic, antibacterial and antifungal activity along with cytotoxicity measurements (ESI Tables 4-6†). As for hexa-bassianolide, antiplasmodial, antimycobacterial and cytotoxic properties were already shown. ${ }^{30,31}$ Additionally, hexabassianolide was found to be active against the parasites Trypanosoma b. rhodesiense, Trypanosoma cruzi and Leishmania donovani (ESI Table $4 \dagger$ ). Most remarkably, both hybrid octa-CDPs showed a significantly increased antiparasitic activity (ESI Table $4 \dagger$ ). Against $T$. cruzi, octa-enniatin $\mathrm{B}$ and octa-beauvericin showed up to 12 -fold lower $\mathrm{IC}_{50}(1.76 \mu \mathrm{M}$ and $0.54 \mu \mathrm{M}$, respectively) than the medical reference drug benznidazole $(6.53 \mu \mathrm{M})$. Furthermore, they are superior to their corresponding natural hexa-CDPs enniatin $\mathrm{B}(2.31 \mu \mathrm{M})$ and beauvericin $(0.76 \mu \mathrm{M})$, respectively. Against $L$. donovani, octa-enniatin B and octa-beauvericin showed up to $>8$-fold lower IC $_{50}(0.07 \mu \mathrm{M}$ and $0.14 \mu \mathrm{M}$, respectively) compared to the medically used reference drug miltefosine $(0.58 \mu \mathrm{M})$. In comparison to enniatin $\mathrm{B}$ and beauvericin $\left(\mathrm{IC}_{50} 0.72 \mu \mathrm{M}\right.$ and
$0.31 \mu \mathrm{M}$, respectively), both compounds were also significantly more active and showed a similar cytotoxicity against rat-derived $L 6$ cells (ESI Table $4 \dagger$ ).

\section{Multiple swapping of fungal CDP synthetases}

With the assigned synthetase functions for precursor and ring size determination in hand, the fungal iterative NRPSs can be seen as a modular assembly platform for a tailored construction of new CDP structures. In order to test the flexibility of fungal CDP synthetases even further, we assessed the influence of multiple domain swaps on hybrid CDP synthesis. For this purpose, we constructed a set of synthetase chimeras for each of the three hybrid CDPs comprising parts from up to three CDP synthetases. All of these six multiple hybrids (EnSYN-BaM 2, BaSYN-EnM $_{2}$, BaSYN-BeM $_{2}$, BeM $_{1}-$ BaM $_{2}-$ EnTC $_{3}$, $\mathrm{EnM}_{1}-\mathrm{BeM}_{2}-\mathrm{BaTC}_{3}, \quad \mathrm{BeM}_{1}-\mathrm{EnM}_{2}-\mathrm{BaTC}_{3}$ ) were capable of producing the predicted hexa- or octa-CDPs in $E$. coli co-expressing NpgA (Fig. 5a). These results demonstrate the first successful multiple cross-pathway assembly in fungal iterative NRPSs. 
Since bacterial NRPS-systems do not tolerate this type of swapping well, ${ }^{13}$ we further examined whether fungal CDP synthetases behave in a similar fashion. For that purpose, we determined the production titers of all wild-type and hybrid CDP synthetases producing hexa-bassianolide. The titers of all singly swapped hybrids $\left(0.03-0.88 \mathrm{mg} \mathrm{L}^{-1}\right)$ remained in the same range as that of wild-type EnSYN $\left(0.15 \mathrm{mg} \mathrm{L}^{-1}\right)$ and BaSYN (0.06 $\left.\mathrm{mg} \mathrm{L}^{-1}\right)$, with the exception of BaSYN-EnC $3\left(24 \mathrm{mg} \mathrm{L}^{-1}\right)$.

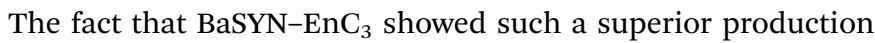
in comparison to BaSYN-EnTC $3\left(0.88 \mathrm{mg} \mathrm{L}{ }^{-1}\right)$ highlights the influence of artificial $\mathrm{T}-\mathrm{C}$ domain pairings in hybrid synthetases, which have a crucial role, i.e. in ester bond formation and macrocyclization. Surprisingly, the hybrid synthetases containing multiple swaps (EnSYN-BaM 2, BeM $\left._{1}-\mathrm{BaM}_{2}-\mathrm{EnTC}_{3}\right)$ showed an enhanced production up to a factor of 520 (55$\left.78 \mathrm{mg} \mathrm{L}^{-1}\right)$ compared to the wild-type EnSYN (0.15 $\left.\mathrm{mg} \mathrm{L}^{-1}\right)$, despite having more heterologous interfaces for domaindomain interactions. This is in stark contrast to virtually all previous studies on module and domain exchanges in bacterial NRPS systems suffering from a significant drop in product titers. $^{32}$ Notably, the relatively high titer was achieved in the bacterial heterologous host $E$. coli, which previously rendered low production yields. ${ }^{17}$ Fungal NRPS systems might thus represent a viable alternative as an engineering platform with promising potential. Furthermore, the results suggest that the efficiency of the biosynthesis is influenced by the N-terminal part of the synthetase. This is supported by the fact that the hybrid EnSYN-BaM ${ }_{2}$ contains a homologous module 1 and $\mathrm{T}_{2 \mathrm{~b}} \mathrm{C}_{3}$, thus pointing to highly important inter-modular interactions like the proposed $\mathrm{C}_{3}-\mathrm{T}_{1}$ interaction during ester bond formation. ${ }^{10}$ However, the highest production was achieved with a heterologous $\mathrm{C}_{3}-\mathrm{T}_{1}$ interface $\left(\mathrm{BeM}_{1}-\mathrm{BaM}_{2}-\mathrm{EnTC}_{3}\right)$. The assumption of a readily exchangeable "bio-brick" system thus appears somewhat simplistic for a sustainable engineering approach.

\section{Conclusions}

Fungal CDP synthetases produce six- and eight-membered CDPs with a broad range of pharmacologically relevant bioactivities. Based on their modular structure, high homology and manageable size, these nonribosomal assembly lines bear good prospects to be an effective engineering platform for the production of novel CDP compounds. Still, a tailored engineering of CDP synthetases is somewhat limited by our poor understanding of the biosynthesis mechanisms. The systematic investigation of the individual contributions of domains to the catalytic activity and synthesis products in this study allow us to confirm the following model: the C-terminal $\mathrm{C}_{3}$ domain performs macrocyclization and determines the ring size independently from $\mathrm{C}_{1}$. In contrast to recent findings, ${ }^{10}$ truncated synthetases lacking the $\mathrm{C}_{1}$ domain are indeed functional in vivo. However, since the absence of $\mathrm{C}_{1}$ significantly affects the production yields of CDPs (drop of synthesis to 1-55\%), a stabilizing and/or supportive role of $\mathrm{C}_{1}$ in the catalytic cycle is apparent. In accordance with a recent crystal structure comprising the $\mathrm{C}-\mathrm{A}-\mathrm{T}-\mathrm{Te}$ domains of the bacterial NRPS
$\mathrm{AB} 3403,{ }^{28}$ the $\mathrm{C}_{1}$ domain may serve as a resting site during precursor adenylation in the $\mathrm{A}_{1}$ domain. This stabilization may also apply to the $\mathrm{A}_{1}$ domain itself, since the $\mathrm{C}$ domains can affect the tertiary structure ${ }^{33}$ and substrate specificities of their downstream A domain partners. ${ }^{34}$

With regard to substrate specificity, various functional hybrid synthetases with a heterologous $\mathrm{C}_{3}$ domain demonstrated that the $\mathrm{C}_{3}$ domain tolerates aliphatic as well as aromatic side chains ( $N$-Me-L-Val/Leu/Ile/Phe). Apart from esterification, $\mathrm{C}_{3}$ also inherits the role of a gauge, measuring product chain length. In contrast to recent statements, ${ }^{\mathbf{1 0}}$ this gauge also allows for cyclization of premature depsipeptide chains. Deletion of the Mt domain, affording the corresponding non-methylated peptides, was able to demonstrate that the absence of structure-modulating $N$-methylations is tolerated. As previous Mt domain deletions reportedly only affected $\beta$-methylation of Glu in daptomycin, ${ }^{\mathbf{1 3}}$ our findings represent a rare example of complete in vivo NRP backbone engineering. The construction of a number of artificial CDP synthetases by means of domain swapping yielded hybrid hexa- and octa-CDPs under in vivo conditions. This way, we were able to produce the hybrid CDP hexa-bassianolide at very high titer of $1.3 \mathrm{~g} \mathrm{~L}^{-1}$ as well as new-to-nature octa-enniatin $\mathrm{B}\left(4 \mathrm{mg} \mathrm{L}^{-1}\right)$ and octabeauvericin $\left(10.8 \mathrm{mg} \mathrm{L}^{-1}\right)$ by using $A$. niger as a robust and cognate heterologous host. While previous approaches with the daptomycin synthetase have shown that multiple domain swaps lead to reduced product yields, ${ }^{13}$ we could significantly enhance CDP production in vivo by constructing functional hybrid synthetases from three different NRPSs. The results highlight the potential of fungal NRPS systems as engineering platforms as well as the crucial role of domain-domain and modulemodule interfaces in NRPS efficiency.

Swapping of parts of the $\mathrm{C}_{3}$ subdomain uncovered functional aspects of macrocyclization and ring size control. According to the bacterial Te mechanism, macrocyclization by the fungal $\mathrm{C}_{3}$ domain is performed when the free-standing hydroxy group at the tail of the linear depsipeptidyl chain is in close proximity to $\mathrm{His}_{\text {cat }}$ of $\mathrm{C}_{3}$ and the substrate thioester. In consequence, CDP ring size probably depends on the size and composition of the cyclization pocket formed by $\mathrm{C}_{3 \mathrm{NTD}}$ and $\mathrm{C}_{3 \mathrm{CTD}}$, along with a competitive process between the elongation and cyclization reaction. This assumption is supported by our observations that, unlike the octa-CDP-producing synthetases, all hexa-CDPgenerating hybrids almost completely lost the capability of producing the cognate eight-membered ring. One reason might be the steric demand of a linear octa-depsipeptide, which may not fit well into the cyclization pocket of a hexa-CDP-producing synthetase. In addition to steric restrictions imposed by the (heterologous) cyclization pocket, ring size may also depend on intrinsic conformational properties of the growing depsipeptide chain. The nature of side chains, and in particular of backbone $N$-methylation, dictates the available conformational space of the depsipeptide backbone and thus affects cyclization efficiencies. For example, Ramachandran plots for peptides comprising $N$-Me-AAs demonstrate the restricted conformational freedom compared to their non-methylated analogs. ${ }^{35}$ However, the distinct mechanism of CDP ring size 
determination remains to be elucidated. By confirming recent findings of CDP production in synthetase variants lacking Ppant $_{2 \mathrm{a} / \mathrm{b}}$, our data also give strong support to the so-called "linear" CDP biosynthesis model. From a formal aspect, employing an alternating upstream and downstream elongation, this NRPS type might be reclassified as belonging to the "complex-type"32 rather than simply linear or iterative. We therefore suggest the term "looping mode", implicating the substrate shuttling between two $\mathrm{C}$ domains in a consecutive manner. Still, since the role of the $\mathrm{T}_{2 \mathrm{~b}}$ domain remains unclear and odd-numbered $\mathrm{T}$ domain-bound intermediates are yet to be observed, ${ }^{8}$ neither of the two models can be completely ruled out. Hence, further information must be gathered with synthetase mutants, e.g. stalled at certain biosynthesis steps, as it has been applied to the bacterial balhimycin synthetase $\mathrm{e}^{36}$ and rifamycin polyketide synthase. ${ }^{37}$

Finally, our experiments show that the design of biocombinatorial swaps in NRPSs can serve well for the synthesis of compounds with enhanced bioactivities. Accordingly, the bioactivity profiling of octa-enniatin B and octa-beauvericin rendered a significantly stronger antiparasitic activity against T. cruzi (up to 12-fold) and L. donovani (up to 8.3-fold) compared to the reference drugs benznidazole and miltefosine, respectively, accompanied by similar cytotoxicity against $L 6$ cells. Our findings give distinct clues for the active and applicable reprogramming of both iterative and linear NRPS assembly lines in further protein engineering approaches. In the future, we aim to expand the existing range of CDP ring sizes beyond the natural hexa- and octa-CDPs, giving rise to a whole class of new-to-nature cyclic fungal products with superior pharmacological activities and efficiencies.

\section{Conflicts of interest}

There are no conflicts of interest to declare.

\section{Acknowledgements}

We thank A. Mühlenweg and A. Poch for the construction of the PPTase-vectors and the Ppant knock-out mutants as well as S. Grätz for synthesizing the D-Hiv precursor. We also express our gratitude to C. Mudalungu for her help with NMR analysis as well as S. Böcker for the construction of pJET_BaSYN and for assisting the transformation of $A$. niger along with T. Schütze. We thank M. Cal, S. Keller-Märki and R. Rocchetti for their help with the parasite assays. KV wishes to thank C. Weigel for excellent technical help in the antimicrobial activity tests. We thank P. Durkin for proofreading of the manuscript.

\section{Notes and references}

1 R. D. Süssmuth, J. Müller, H. von Döhren and I. Molnár, Nat. Prod. Rep., 2011, 28, 99-124.

2 S. C. Feifel, T. Schmiederer, T. Hornbogen, H. Berg, R. D. Süssmuth and R. Zocher, ChemBioChem, 2007, 8, 1767-1770.
3 J. Müller, S. C. Feifel, T. Schmiederer, R. Zocher and R. D. Süssmuth, ChemBioChem, 2009, 10, 323-328.

4 D. Matthes, L. Richter, J. Müller, A. Denisiuk, S. C. Feifel, Y. Xu, P. Espinosa-Artiles, R. D. Süssmuth and I. Molnár, Chem. Commun., 2012, 48, 5674.

5 M. Krause, A. Lindemann, M. Glinski, T. Hornbogen, G. Bonse, P. Jeschke, G. Thielking, W. Gau, H. Kleinkauf and R. Zocher, J. Antibiot., 2001, 54, 797-804.

6 N. Madry, R. Zocher, K. Grodzki and H. Kleinkauf, Appl. Microbiol. Biotechnol., 1984, 20, 83-86.

7 R. Zocher, U. Keller and H. Kleinkauf, Biochem. Biophys. Res. Commun., 1983, 110, 292-299.

8 W. Weckwerth, K. Miyamoto, K. Iinuma, M. Krause, M. Glinski, T. Storm, G. Bonse, H. Kleinkauf and R. Zocher, J. Biol. Chem., 2000, 275, 17909-17915.

9 M. Glinski, C. Urbanke, T. Hornbogen and R. Zocher, Arch. Microbiol., 2002, 178, 267-273.

10 D. Yu, F. Xu, S. Zhang and J. Zhan, Nat. Commun., 2017, 8, 15349.

11 X. Gao, S. W. Haynes, B. D. Ames, P. Wang, L. P. Vien, C. T. Walsh and Y. Tang, Nat. Chem. Biol., 2012, 8, 823-830.

12 H. D. Mootz, D. Schwarzer and M. A. Marahiel, Proc. Natl. Acad. Sci. U. S. A., 2000, 97, 5848-5853.

13 K. T. Nguyen, D. Ritz, J.-Q. Gu, D. Alexander, M. Chu, V. Miao, P. Brian and R. H. Baltz, Proc. Natl. Acad. Sci. U. S. A., 2006, 103, 17462-17467.

14 D. Butz, T. Schmiederer, B. Hadatsch, W. Wohlleben, T. Weber and R. D. Süssmuth, ChemBioChem, 2008, 9, 1195-1200.

15 H. D. Mootz, N. Kessler, U. Linne, K. Eppelmann, D. Schwarzer and M. A. Marahiel, J. Am. Chem. Soc., 2002, 124, 10980-10981.

16 D. Yu, F. Xu, D. Gage and J. Zhan, Chem. Commun., 2013, 49, 6176.

17 S. Zobel, S. Boecker, D. Kulke, D. Heimbach, V. Meyer and R. D. Süssmuth, ChemBioChem, 2016, 17, 283-287.

18 J. W. A. van Dijk, C. Guo and C. C. C. Wang, Org. Lett., 2016, 18, 6236-6239.

19 L. Richter, F. Wanka, S. Boecker, D. Storm, T. Kurt, Ö. Vural, R. Süssmuth and V. Meyer, Fungal Biol. Biotechnol., 2014, 1, 4.

20 S. A. Samel, G. Schoenafinger, T. A. Knappe, M. A. Marahiel and L.-O. Essen, Structure, 2007, 15, 781-792.

21 M. Biasini, S. Bienert, A. Waterhouse, K. Arnold, G. Studer, T. Schmidt, F. Kiefer, T. G. Cassarino, M. Bertoni, L. Bordoli and T. Schwede, Nucleic Acids Res., 2014, 42, W252-W258.

22 J. Zhang, N. Liu, R. A. Cacho, Z. Gong, Z. Liu, W. Qin, C. Tang, Y. Tang and J. Zhou, Nat. Chem. Biol., 2016, 12, 1001-1003.

23 A. Tanovic, S. A. Samel, L.-O. Essen and M. A. Marahiel, Science, 2008, 321, 659-663.

24 E. Conti, T. Stachelhaus, M. A. Marahiel and P. Brick, EMBO J., 1997, 16, 4174-4183.

25 M. A. Marahiel, T. Stachelhaus and H. D. Mootz, Chem. Rev., 1997, 97, 2651-2674.

26 D. Lücke, T. Dalton, S. V. Ley and Z. E. Wilson, Chem.-Eur. J., 2016, 22, 4206-4217. 
27 S. Supothina, M. Isaka, K. Kirtikara, M. Tanticharoen and Y. Thebtaranonth, J. Antibiot., 2004, 57, 732-738.

28 E. J. Drake, B. R. Miller, C. Shi, J. T. Tarrasch, J. A. Sundlov, C. Leigh Allen, G. Skiniotis, C. C. Aldrich and A. M. Gulick, Nature, 2016, 529, 235-238.

29 Y. A. Ovchinnikov, V. T. Ivanov, I. I. Mikhaleva and M. M. Shemyakin, Izv. Akad. Nauk SSSR, Ser. Khim., 1964, 581, 1912.

30 C. Nilanonta, M. Isaka, R. Chanphen, N. Thong-orn, M. Tanticharoen and Y. Thebtaranonth, Tetrahedron, 2003, 59, 1015-1020.

31 A. A. Sy-Cordero, C. J. Pearce and N. H. Oberlies, J. Antibiot., 2012, 65, 541-549.
32 R. D. Süssmuth and A. Mainz, Angew. Chem., Int. Ed., 2017, 56, 3770-3821.

33 M. J. Tarry, A. S. Haque, K. H. Bui and T. M. Schmeing, Structure, 2017, 25, 783-793.

34 S. Meyer, J.-C. Kehr, A. Mainz, D. Dehm, D. Petras, R. D. Süssmuth and E. Dittmann, Cell Chem. Biol., 2016, 23, 462-471.

35 T. T. Tran, H. Treutlein and A. W. Burgess, Protein Eng., Des. Sel., 2006, 19, 401-408.

36 D. Bischoff, B. Bister, M. Bertazzo, V. Pfeifer, E. Stegmann, G. J. Nicholson, S. Keller, S. Pelzer, W. Wohlleben and R. D. Süssmuth, ChemBioChem, 2005, 6, 267-272.

37 A. Stratmann, C. Toupet, W. Schilling, R. Traber, L. Oberer and T. Schupp, Microbiology, 1999, 145, 3365-3375. 